\title{
Nearby Young Moving Groups: Statistical Methods and Challenges for Assigning Membership
}

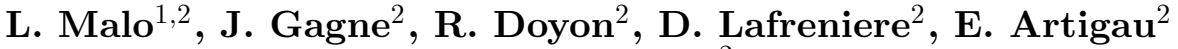 \\ and L. Albert ${ }^{2}$ \\ ${ }^{1}$ Canada-France-Hawaii Telescope, 65-1238 Mamalahoa Hwy, Kamuela Hawaii, 96743, USA. \\ email: malo@cfht.hawaii.edu \\ ${ }^{2}$ Institut de Recherche sur les Exoplanetes (iREx), Universite de Montreal, Departement de \\ Physique, C.P. 6128 Succ. Centre-ville, Montreal, QC H3C 3J7, Canada.
}

\begin{abstract}
Young associations, being sparsely populated and relatively close to the Sun, their members are found all over the sky. In the Solar Neighborhood, young moving groups are found within $100 \mathrm{pc}$ with ages ranging from 5 to $120 \mathrm{Myr}$. While known members of these groups were identified mostly through the Hipparcos data, only the most massive members have been fully characterized so far, and defined the core members. In the last decades, several new candidate members have been identified, using different approaches. Based on the global properties of the core members (kinematics and over luminosity), those methods used several criteria to establish the membership, from qualitative manner to quantitive methods using reduced chisquared or membership probability. A full confirmation of the membership for those numerous candidates requires radial velocity and parallax measurements to confirm their kinematics, agedating indicator measurement to assess their youth and multiplicity follow-up to rule out binary objects. In this proceeding, we summarize a general recipe to assign membership, describe the numerous challenges for assigning membership, and end with a discussion on the appropriateness and reliability of the BANYAN I and II tools to assess membership.
\end{abstract}

Keywords. methods: statistical,(Galaxy:) solar neighborhood, stars: low-mass, stars: pre-main sequence, techniques: radial velocities.

\section{Introduction}

Nearby young comoving groups (NYMG) are sparse, gravitationally unbound stellar associations comprising a few dozens of stars scattered within $\sim 100$ pc of the Sun with ages ranging from 5 to a few hundred Myr. Comoving group members are characterized by a common position and space motion within the Galaxy. As a result of a projection effect, they display an organized motion in the sky moving toward a convergent point and this can be used to discriminate genuine members from field stars. Since 1958, several methods have been developed to identify new associations and/or new members of a given association (Eggen 1958, Montes et al. 2001, Zuckerman \& Song 2004, Torres et al. 2006). Moreover, Hipparcos mission combined with multiple radial velocity studies led to the identification of the most massive members of the NYMGs, but relatively shallow completeness magnitude prevented the detection of low-mass members. Assuming that NYMG stars follow a typical Initial Mass Function, the vast majority of low-mass members are expected to have gone unnoticed. This led to a number of efforts to extend measurements of proper motion, radial velocity and parallax to the low-mass regime (e.g., Shkolnik et al. 2012). To complete the census of NYMG members, kinematics alone is 
not enough to confirm membership, independent youth indicators are also mandatory to confirm the age of these stars.

\section{General recipe to assign membership}

Identifying new members of NYMGs requires to be careful on the selection of the core members, as well as the determination of their global properties and finally the method used to quantify the membership. The following section summarizes the key elements for each of the essential steps.

\subsection{Reference sample}

For the sole purpose of establishing the core membership of the association, one needs to identify strong members (bona fide). Stars should be considered bona fide members of young kinematic groups only when a good measurement of trigonometric distance, proper motion and radial velocity are available, which means that Galactic space velocity and Galactic positions are accurate. Moreover, bona fide members need to display youth indicators which depend on the mass and age, and finally an appropriate location in the HR diagram. Several studies have established lists of bona fide members, such as Zuckerman \& Song (2004), Torres et al. (2008), Fernandez et al. (2008).

\subsection{Common properties}

Because of the coeval formation of a given association, all members share, to within a few $\mathrm{km} \mathrm{s}^{-1}$, a common space velocity within the Galaxy. The galactic space motion of a star, $U V W$, is determined from its sky position $(\alpha, \delta)$, radial velocity (RV), proper motion, and parallax, using the Johnson \& Soderblom (1987) relations.

By virtue of their youth and their coeval formation, the members of young associations have had little time to disperse within the Galaxy. As a result, the positions $X Y Z$ of the members of a young association are relatively well confined within the Galaxy. The galactic position of a star, $X Y Z$, is determined from its sky position $(\alpha, \delta)$ and trigonometric distance.

Color-Magnitude Diagrams (CMD) have been a crucial tool for identifying young stars, since they are over-luminous compared to an old population. The color indices used are strongly mass dependant. To discern between young and old low-mass stars wide color indices (e.g. $V-K, I_{c}-J$ ) are good to provide protection against measurement errors and time variability (Zuckerman \& Song 2004). Figure 1 shows CMD for low-mass stars using $M_{J}$ vs $I_{c}-J$. Past studies have shown that $B-V$ can be useful for more massive stars (Zuckerman \& Song 2004) and color indices in the near and mid-infrared should be used for brown dwarfs and planetary-mass objects (Gagne et al. 2014).

\subsection{Metric}

A key element in order to quantitatively assess star membership in a given NYMG is confirmed if candidate's $U V W X Y Z$ match those of a given NYMG. When radial velocity and parallax are available, one can minimize a reduced $\chi^{2}$ statistic with several degrees of freedom to quantify the match. This method has been used by Shkolnik et al. (2012), Faherty et al. (2012), Riedel et al. (2014) using the full 6 dimensions ( $U V W X Y Z$ ) and applied to 14 NYMGs and young associations.

When radial velocity and parallax are not available, one has to predict the values, which a star should have if it was an actual member of a given NYMG. To predict the radial velocity value, Zuckerman \& Song (2004) have developed the good $U V W$ box method which determines the radial velocity required combined with the distance and 


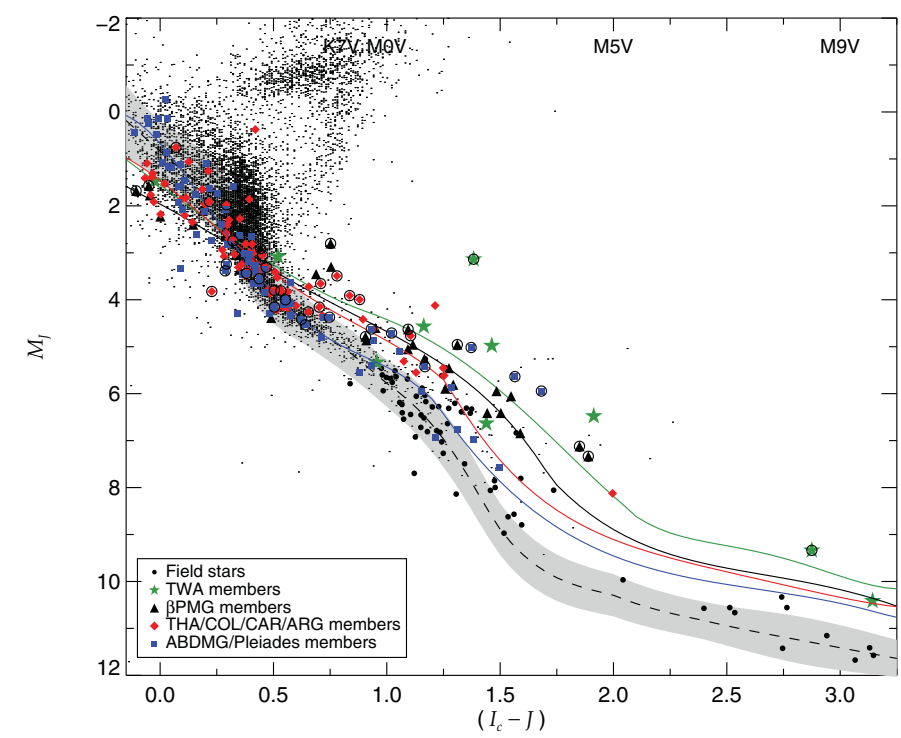

Figure 1. Color-magnitude diagram $\left(M_{J}\right.$ vs $\left.I_{c}-J\right)$ for members of TWA (green stars), $\beta$ PMG (black triangles), THA, COL, CAR, and ARG (red diamonds), and ABDMG and Pleiades (blue squares). Fields stars are represented by dots and filled black circles, and binary stars are those with black circles superposed on their own symbol (Malo et al. 2013).

sky position, to match a given NYMG's galactic space velocity. Moreover, Rodriguez et al. (2013) have developed GALNYSS method which includes the convergent point method to derive the radial velocity and kinematic distance for a given NYMG.

To predict the distance, Torres et al. (2006) have developed the SACY project, based on the minimization of a merit function to infer the distance to the star. This merit function requires to match the $U V W X Y Z$ and the expected brightness as a function of color index.

A more robust method, combining all previous works, has been developed by Malo et al. (2013) and Gagné et al. (2014), by building a kinematic model of a given NYMG to predict the amplitude of the proper motion and radial velocity which a star should have if it were a member of the NYMG. The kinematic model is combined with a Bayesian analysis to predict the most likely distance to the star. Therefore, the Bayesian Analysis for Nearby Young AssociatioNs (BANYAN) tool derives membership probabilities by combining Bayesian inference to empirical models for seven observables characterizing the kinematics $(U V W X Y Z)$ and photometry of known members. Such models were built for the seven closest and youngest well-defined moving groups : TW Hydrae, $\beta$ Pictoris, Tucana-Horologium, Columba, Carina, Argus and AB Doradus. Also, models were built for a field sample of stars allowing for each candidate member, kinematic and photometric comparisons to an old field population. In addition to the membership probability, statistical predictions for radial velocity and distance, BANYAN includes a binary hypothesis, which should be interpreted as an over-luminosity compared to other young stars, which could be due to the presence of a binary companion, chromospheric activity, or peculiar colors of the star.

More recently, Gagné et al. (2014) developed BANYAN II, which includes a more sophisticated kinematic model (ellipsoids with arbitrary rotation) and uses NIR colormagnitude diagrams to specifically target the identification of brown dwarfs and planetary-mass objects in these moving groups. Moreover, expected field and moving 


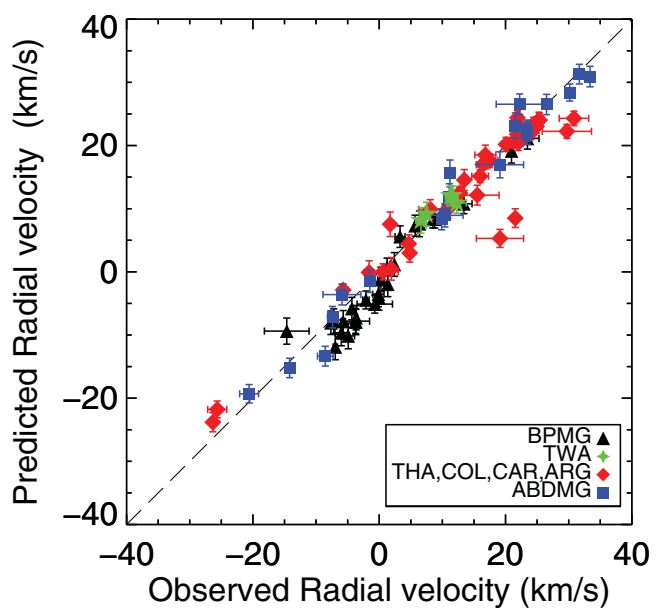

Figure 2. Comparison between predicted and observed radial velocities for the 111 candidate members ( $\mathrm{Pv}>90 \%$ and excluding known binaries) (Malo et al. (2014)).

group populations are taken into account as prior probabilities, and an extensive falsepositive and hit rate analysis is performed based on a Besançon Galactic model (Robin et al. in prep).

A web-based tool of the Bayesian techniques are available at :

www.astro.umontreal.ca/ malo and www.astro.umontreal.ca/ gagne .

\subsection{Membership confirmation}

We used the BANYAN I and BANYAN II tools to newly identify 200 K5-M5, 175 M4L7 candidate members to NYMGs, and 39 previously known brown dwarfs with signs of youth (Gagne et al. 2014, Gagne et al. in press). Although the analysis unveiled new highly probable candidate members to NYMGs, a word of caution is necessary before assigning a firm membership to these objects. These candidates will be attributed the status of bona fide members only after their radial velocity and parallax are measured and shown to be consistent with the radial velocity and statistical distance predicted by our analysis. For this reason, Malo et al. (2014) presented RV measurements for 219 K7M5 candidate members, using high resolution spectroscopy. This follow-up study shows that the predicted radial velocity agrees with the measured value for 130 stars. Figure 2 shows the comparison between predicted and observed values.

Furthermore, a collaboration with A. Riedel (Riedel et al. 2014) and the CTIOPI group, as well as recent parallax measurements in the literature (Dupuy \& Liu 2012; Shkolnik et al. 2012; Liu et al. 2013; Dittmann et al. 2014) confirmed statistical distance predictions from BANYAN for 30 candidate members.

In order to confirm the youth of the candidates, signs of youth depending on the spectral type should be measured. For solar type stars, coronal activity indicators such as X-ray and UV emission are often used (Soderblom 2010). For low-mass dwarfs, chromospheric and coronal activity indicators, lithium abundance and rotation are common youth indicators. In the case of brown dwarfs, spectroscopic indicators of low-gravity are preferred, such as the equivalent width of Na I, KI, the depth of VO band-heads, as well as the shape of the H-band continuum.

Last, because the interpretation of the observed luminosity is different in the case of an unresolved multiple system, RV monitoring and high contrast imaging should be pursued 
to identify binary systems within the proposed bona fide members, before claiming final candidate membership.

\section{Challenges}

Assigning candidate membership in NYMGs is a complex puzzle, which requires huge amount of tools and knowledge. There are at least three challenges which we faced when identifying and confirming candidate membership. First, quantitative assessment requires a precise determination of the global properties for NYMG members, which are strongly dependant on the method used and the sample of bona fide members. As shown in Gagne et al. (2014), parametrization of $U V W X Y Z$ using ellipsoids can better reproduce the shape of the clusters.

A second challenge when assigning membership is the missing radial velocity and parallax information, for the vast majority of the candidates. Given that one needs to develop such tools to predict radial velocity and parallax values which a star should have if the star was a member of a given NYMG. Furthermore, these values have to be measured with a high precision and should validate the predictions.

Finally, independently of the method used to assess membership, a contamination rate simulation should be performed and the contamination rate should be taken into account, when claiming the final candidate membership. To minimize the contamination rate, analysis methods should use star's kinematics ( $U V W X Y Z)$ complemented with the photometric properties (over-luminosity) and analysis methods should include not only NYMGs but also an old field population for comparison. BANYAN tools have performed such contamination analysis showing false alarm rate between $5-15 \%$ depending on the type of stars and NYMGs considered (Malo et al. 2013, Gagne et al. 2014)

\section{Future prospects}

The main limitation of the current BANYAN tool is that it does not include young association members located beyond 100 pc such as Eta Cha (Zuckerman \& Song 2004), the Scorpius-Centaurus complex (de Zeeuw et al. 1999) and the Pleiades (Jeffries 1995). The main reason for this is that the construction of reliable spatial and kinematic models for these associations requires precise measurements of RV and distance for a significant set of credible members. Those RV and parallax measurements are still not available. However, Rizzuto et al. (2011), Rizzuto et al. (2012) have been focusing on combining bayesian analysis and global properties of Scorpius-Centaurus complex members, resulting in strong candidate members, for which predicted values will be available soon. The Gaia mission will provide such an opportunity by providing measurements of trigonometric distance for objects with $V \leqslant 20$. However, RV measurements from Gaia mission will be limited to a precision of a few $\mathrm{kms}^{-1}$ for faint low-mass stars and brown dwarfs. This brings out the important role that ground-based RV surveys reaching precisions of less than $1 \mathrm{kms}^{-1}$ will play in characterizing the kinematics of red objects. With accurate RV and parallax measurements for all stars in hand, the determination of the $U V W, X Y Z$ for young group members beyond $100 \mathrm{pc}$ will become possible. Moreover, the discovery of new NYMGs within 100 pc will be now possible, helping with a better understanding of the solar neighborhood formation. Current and next generation instruments such as GRACES (Chene et al. 2014) at Gemini-North, SPIRou at CFHT (Delfosse et al. 2013) and CARMENES (Quirrenbach et al. 2014) are opening the door to precise radial velocity measurements leading to more accurate galactic space velocities, when combined with Gaia parallaxes, which are required to identify new NYMGs in the Solar Neighborhood. 


\section{Acknowledgement}

The authors would like to thank the organizers of the Young Stars Near the Sun meeting, for providing the opportunity to have this full day discussion about how to identify and confirm star's membership; and the participants for engaging in a productive discussion. We thank our collaborators: Jackie Faherty, Adric Riedel, Kelle Cruz and Gregory Feiden. This work was supported through grants from the Canada-France-Hawaii Telescope, the Fond de Recherche Quebecois - Nature et Technologie and the Natural Science and Engineering Research Council of Canada.

\section{References}

Chene, A-N., Padzer, J., Barrick, G., et al. 2014, in SPIE, 9151, id. 915147

de Zeeuw, P. T., Hoogerwerf, R., de Bruijne, J. H. J., Brown, A. G. A., \& Blaauw, A. 1999, AJ, 117,354

Delfosse, X., Donati, J.-F., Kouach, D., et al. 2013, in SF2A-2013: Proceedings of the Annual meeting of the French Society of Astronomy and Astrophysics, ed. L. Cambresy, F. Martins, E. Nuss, \& A. Palacios, 497

Dittmann, J. A., Irwin, J. M., Charbonneau, D., \& Berta-Thompson, Z. K. 2014, AJ, 784, 156

Dupuy, T. J. \& Liu, M. C. 2012, ApJS, 201, 19

Eggen, O. J. 1958, MNRAS, 118, 65

Faherty, J. K, Burgasser, A. J, Walter, F. M, et al. 2012, ApJ, 752, 56

Fernandez, D., Figueras, F., \& Torra, J. 2008, A $6 A$, 480, 735

Gagné, J., Lafrenière, D., Doyon, R., Malo, L., \& Artigau, É. 2014, ApJ, 783, 121

Jeffries, R. D. 1995, MNRAS, 273, 559

Johnson, D. R. H. \& Soderblom, D. R. 1987, AJ, 93, 864

Liu, M. C., Dupuy, T. J., \& Allers, K. N. 2013, AN, 334, 1

Malo, L., Doyon, R., Lafrenière, D., et al. 2013, ApJ, 762, 88

Malo, L., Artigau, É., Doyon, R., et al. 2014, ApJ, 788, 81

Montes, D., Lopez-Santiago, J., Galvez, M. C., et al. 2001, MNRAS, 328, 45

Quirrenbach, A., Amado, P. J., Caballero, J. A., et al. 2014, in SPIE, 9147, id 91471F

Riedel, A. R., Finch, C. T., Henry, T. J., et al. 2014, AJ, 147, 85

Rizzuto, A. C., Ireland, M. J., \& Robertson, J. G. 2011, MNRAS, 416, 3108

Rizzuto, A. C., Ireland, M. J., \& Zucker, D. B. 2012, MNRAS, 421, 97

Robin, A. C., Marshall, D. J., Schultheis, M., \& Reylé, C. 2012, A\&GA, 538, A106

Rodriguez, D. R., Zuckerman, B., Kastner, J. H., et al. 2013, AJ, 774, 101

Shkolnik, E. L., Anglada-Escude, G., Liu, M. C., et al. 2012, AJ, 758, 56

Soderblom, David. R. 2010, ARA\&A, 48, 581

Torres, C. A. O., Quast, G. R., da Silva, L., et al. 2006, A\&\&A,460, 695

Torres, C. A. O., Quast, G. R., da Melo, C. H. F., et al. 2008, in Handbook of Star forming Regions, Vol. II: The Southern Sky, ed. B. Reipurth (ASP Monograph Publ., Vol. 5; San Francisco, CA: ASP), 757

Zuckerman, B. \& Song, I. 2004, ARA\&A, 42, 685 